\title{
Les Expressions Phraséologiques Dans La Presse Écrite Française Et Géorgienne
}

\author{
Mariam Pareshishvili, PhD
}

Le Centre de Langues de l'Université géorgienne auprès du Patriarcat

doi: 10.19044/esj.2016.v12n26p410 URL:http://dx.doi.org/10.19044/esj.2016.v12n26p410

\begin{abstract}
The aim of this article is to present the using of phraseological units in Georgian and French newspapers headlines. The role of the newspapers headlines is very important, because, to a certain extent, the understanding of the text depends on how the title presents the underlying text. Consequently, the journalists often use phraseological units to draw the attention of the readers. Phraseological units exist in the language as ready-made units and make newspapers very expressive because of their figurative nature. The analysis of press texts in both languages showed that the phraseological units are used for the purpose of text. We are going to try to show which type of phraseological units are used most of the time in the Georgian and French newspapers headlines.
\end{abstract}

Keywords : Phraseological units, newspapers headlines, background knowledge, news texts

\section{Resume}

Notre communication est consacrée aux unités phraséologiques dans les médias français et géorgien. Le corpus choisi est un corpus d'articles politiques en deux langues. Le choix des textes journalistiques est conditionné par l'évolution rapide de la phraséologie qui se reflète largement dans la presse écrite, ainsi que par la complexité de sa reformulation. La maîtrise de la phraséologie constitue une composante importante de la compétence linguistique $\mathrm{du}$ fait que les textes journalistiques regorgent d'unités phraséologiques. Nous allons essayer d'identifier les unités phraséologiques qui sont utilisées le plus souvent dans la presse écrite française et géorgienne.

Mots clés : Les unités phraséologiques, les intitulants des articles politiques, la presse écrite, la compétence linguistique 


\section{Introduction}

Dans la société actuelle les médias sont largement présents dans la vie quotidienne et constitue une source principale de toute information pour une grande majorité des hommes. Le grand avantage que présentent les médias est d'informer rapidement et d'une manière continue des événements importants qui se produisent au niveau national et international.

La presse écrite est un genre particulier, dont la particularité principale est le reflet momentané de la réalité. Le style des journaux est un style à part, dont les fonctions communicatives sont d'informer et de donner une opinion. Le discours journalistique, qui est un type spécifique de communication a toujours joué un rôle important dans la formation de l'opinion public.

A travers de notre modeste contribution, nous allons essayer d'étudier la fréquence et les occurrences des expressions phraséologiques (surtout des expressions idiomatiques) dans des textes journalistiques français et géorgiens et d'identifier les expressions idiomatiques les plus utilisées par des journalistes dans les journaux les plus populaires.

Comme des journaux touchent des domaines différents, nous avons limité le champ de notre étude en focalisant la réflexion sur les expressions idiomatiques dans les titres des textes à caractère politique afin de préserver une certaine homogénéité. Le corpus de notre étude est constitué d'articles des journaux quotidiens les plus vendus en France et en Géorgie (Le Figaro, Le Monde, La Libération, з3опоl зsmoßns, 24 ussono et de journaux d'information en ligne les plus lus).

Le choix des intitulants des articles journalistiques en tant que l'objet de notre étude a été déterminé par le fait que le titre constitue un facteur important de l'éveil de l'intérêt auprès du public et se caractérise particulièrement par l'abondance des unités phraséologiques. A signaler que les titres constituent une entité sémantiquement complète et polyfonctionnelle. Dans la lecture du texte le titre joue un rôle important. La compréhension ou l'interprétation du texte pourrait dépendre de sa présentation par le titre.

Les expressions idiomatiques sont employées hors de leurs sens propres. Elles donnent de la couleur à notre langue et constituent une des principales composantes de la phraséologie. Les tournures phraséologiques constituent un procédé stylistique particulier. Elles enrichissent le texte en atténuant ou au contraire en accentuant le sens de certains mots de la langue. Le recours aux expressions idiomatiques enrichit le vocabulaire journalistique et permet aux lecteurs de percevoir l'information d'une manière facile et concise. Elles embellissent et rendent les nouvelles plus impressionnantes. En plus, elles constituent un indicateur du statut social des lecteurs car la compréhension littérale des expressions devrait toujours être plus rapide que la compréhension idiomatique. 


\section{Les particularités des textes journalistiques}

Normalement, tout texte journalistique doit être clair et attrayant. La première fonction attribuée à la presse est celle d'informer. Pourtant, la presse écrite a également pour fonction de " convaincre " et "d'influencer » l'opinion publique.

De différents journaux n'abordent pas l'information de la même façon. Les journaux peuvent traiter différemment la même information. Certains journaux ont tendance à interpréter les faits en dramatisant, de façon à accrocher ou choquer le public ou bien à respecter l'orientation de leur média $^{70}$. Pour parvenir à ces objectifs les propos formulés dans le texte sont souvent transposés à l'aide de diverses figures de style visant à produire des effets particuliers.

Effectivement, chaque journal a son propre style. Le lexique de la presse écrite est très varié du point de vue stylistique et des registres. Le langage du discours journalistique n'est pas le même en France et en Géorgie. Contrairement aux titres des articles politiques géorgiens, les titres des articles français respectent certaines normes de l'étique journalistique et ont recours à des titres moins longs et percutants (nous parlons notamment des journaux ayant une notoriété dans leur pays de diffusion : Le Monde, le Figaro) dont le style se diffère de celui de la presse jaune. Par contre, les journalistes géorgiens privilégient les titres percutants. La presse écrite géorgienne presque dans sa totalité (il y a bien évidemment certaines exceptions dans le cas des journaux de haute tenue) est orientée sur le choc. Nombreux sont les cas où les articles ne répondent aucunement à leurs intitulations. La presse géorgienne se caractérise également par un usage excessif des expressions phraséologiques afin d'introduire une intrigue et d'attirer l'attention du lecteur. Les titres aussi accrocheurs nuisent effectivement au sérieux des journaux et à la crédibilité de l'information diffusée.

Les textes journalistiques se caractérisent par un emploi important et marquant des expressions phraséologiques par rapport à d'autres genres de textes et plus particulièrement par l'abondance d'expressions idiomatiques qui occupent un grand espace dans les structures figées. Les expressions idiomatiques ont aussi la fonction de représenter une situation, une idée ou un évènement. Elles sont utilisées pour émouvoir, impressionner, redonner de la vie aux idées exprimées, persuader, retenir l'attention de la personne à qui l'on s'adresse. La notion de la phraséologie est fondamentale pour la compréhension du texte.

\footnotetext{
${ }^{70} \mathrm{http}$ ://ilcea.revues.org/210
} 


\section{Les fonctions des expressions phraséologiques}

Les expressions idiomatiques sont des locutions courantes en français et en géorgien. Leur signification n'est pas le résultat logique de l'addition des significations de ses composantes. Effectivement, ces expressions embellissent la langue et rendent le discours plus éloquent. Beaucoup d'entre elles présentent un caractère métaphorique.

Les expressions ou locutions idiomatiques sont bien nombreuses dans la plupart des langues. Elles sont des locutions figées dont la structure sémantique contient de la connotation $^{71}$. Les expressions idiomatiques comportent des données très importantes sur les valeurs et la culture de la société.

Selon Antonella Capra ${ }^{72}$ les tournures idiomatiques naissent d'une culture commune, sont l'expression d'un savoir universel, sortent directement de la «voix du peuple» : en les utilisant le locuteur est conscient de tout cela, sait pertinemment qu'il n'est pas le créateur de ces énoncés. De plus il se rend aussi compte que son interlocuteur possède les mêmes connaissances et qu'il saura reconnaître l'énoncé figé. Ce qu'il veut prouver donc, ce n'est pas sa capacité à «bien parler », mais plutôt à « bien savoir » : savoir qu'il y a une sagesse populaire, une connaissance partagée avec ses destinataires, qui lui servira à communiquer sa pensée, à convaincre l'autre que son discours est valable, puisque certifié par toute une culture. Savoir qu'il fait partie d'une communauté linguistique, de cette même communauté qui approuve ses propos, semble donner de l'assurance au locuteur. De cette manière, il appuie son discours sur des raisons "irréfutables", il utilise une reconnaissance sociale et se prête à attirer l'attention de son destinataire, pour le persuader de la justesse de ses propos.

Les langues du monde possèdent un grand nombre de locutions idiomatiques qui reflètent des particularités nationales et psychologiques, des particularités de la perception de l'univers par leurs porteurs. Elles constituent un véritable joyau de la culture d'une ethnie ou d'une nation ${ }^{73}$.

Selon Ducrot les El nous donnent des informations fondamentales sur l'organisation conceptuelle du monde environnant, des données du passé et du présent. Les expressions pénètrent dans la vie quotidienne de la langue, elles y trouvent leur essence et elles enrichissent les relations que le sujet parlant établit avec le monde et les hommes. Elles donnent plus d'expressivité à la langue. Intégrées dans le discours, elles établissent entre le locuteur et son interlocuteur une certaine connivence, un terrain d'entente, un savoir partagé,

\footnotetext{
${ }^{71} \mathrm{http}: / /$ www.academia.edu/6362051/Analyse_de_la_frequence_des_expressions_idiomatiques_utilise es_dans_les_articles_de_journaux

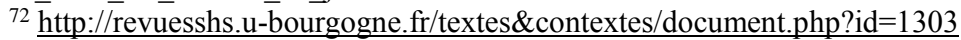

$73 \mathrm{http}: / / \mathrm{www}$. youscribe.com/catalogue/livres/education/manuels-scolaires/dictionnaire-russe-francaisdes-locutions-idiomatiques-equivalentes-176725
} 
qui ne pourra que faciliter la communication. Elles fonctionnent comme un présupposé: «ce qui est commun aux deux personnages d'un dialogue, comme l'objet d'une complicité fondamentale qui lie entre eux les participants de l'acte communicatif $\gg$.

\section{Les expressions phraséologiques dans la presse écrite}

Les expressions idiomatiques sont considérées comme des unités linguistiques qui représentent des groupes de mots souvent imprévisibles dans leur structure et surtout dans leur sens.

Effectivement, les mots sont liés à l'histoire et à la culture d'une communauté linguistique. Pourtant, comme le signale Le Guern, le langage n’est pas «un calque direct de la réalité existante»: il suppose «nécessairement une interprétation intellectuelle».

En général, le discours journalistique se caractérise par 1) un taux élevé

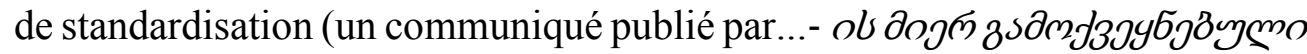
змдубозО; les pourparlers sont en cours entre - додcooбsकुm

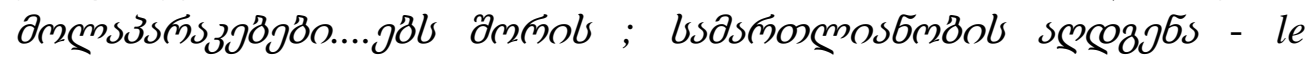

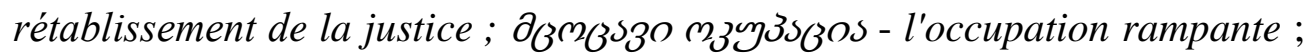
2) l'utilisation fréquente de l'infinitif narratif $(\mathrm{Et}+$ sujet+verbe déclaratif à l'infinitif) «Et le journal de titrer: "la bataille est lancée." - "סळдmens

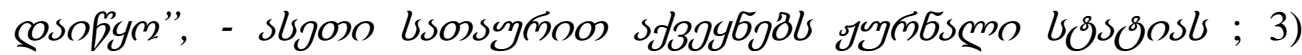
l'utilisation du discours indirect, 4) la présence de procédés de style (la métaphore, la métonymie) et des realia politico-culturels : Le palais de l'Élysée

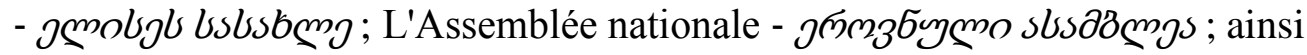
que par l'abondance des expressions phraséologiques. Naturellement, pour influencer l'opinion publique les journalises tentent d'utiliser les moyens les plus marquants y compris les unités phraséologiques.

L'analyse des titres des articles politiques en deux langues nous montre que les expressions et les locutions idiomatiques sont prédominantes dans la presse écrite, les proverbes et les dictons, sont utilisés moins rarement dans les énoncés intitulants ( $\mathrm{La}$ meilleure défense, c'est 1'attaque- bsy3jonglm

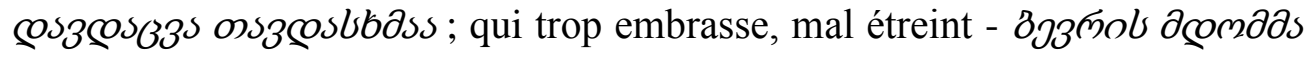
उMOSO coszsngsm; Tel qui rit vendredi, dimanche pleurera - bogomb

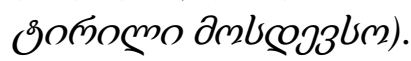

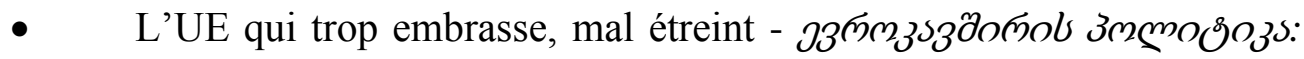

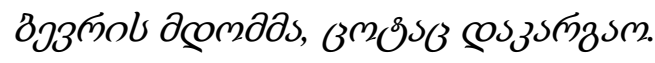




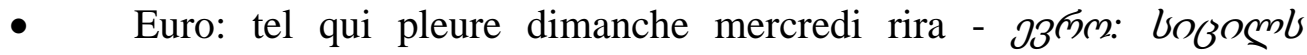

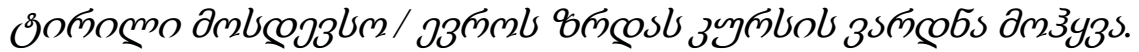

- $\quad$ Qui vivra verra si les jours du régime syrien sont comptés - @oßm

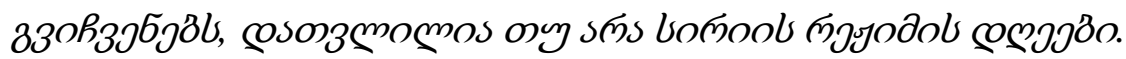

Le discours journalistique français et géorgien abonde en expressions

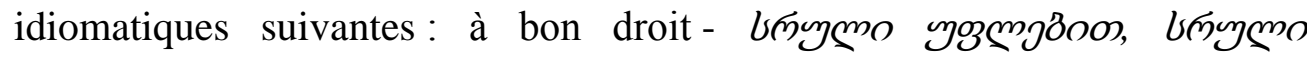

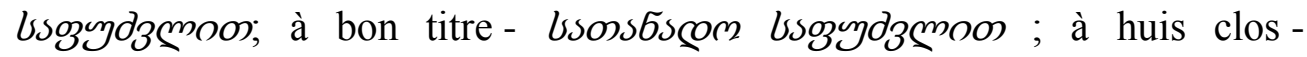

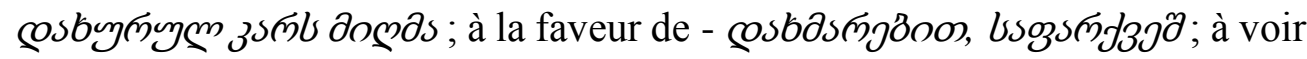
avec (rien à voir, pas beaucoup à voir...) lughonml fmbs ; aller de pair avec -

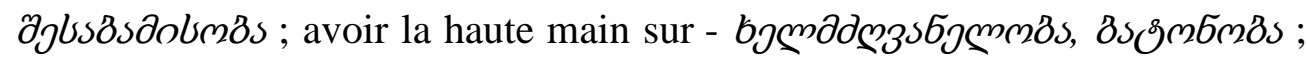

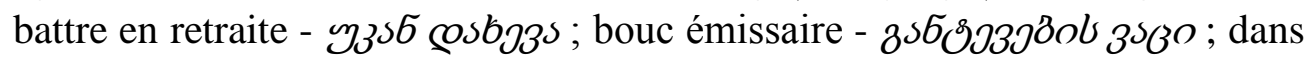
le cadre de -ol gsım

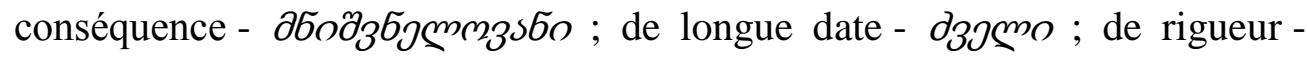

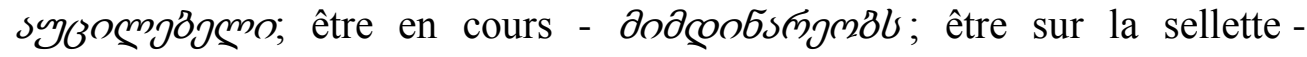

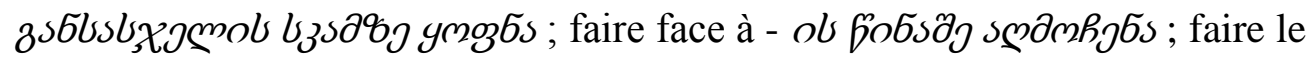

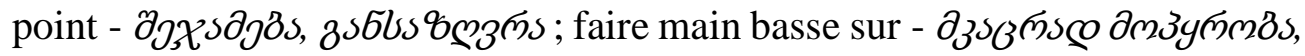

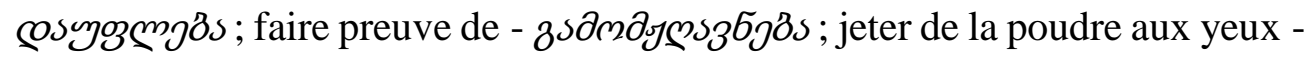

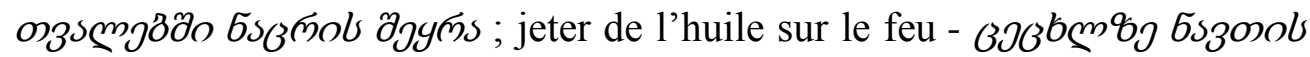
cosbbas ; lettre morte - jsssmsool 6szmJzo ; l'ordre du jour - copol

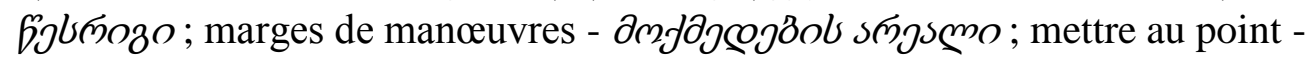

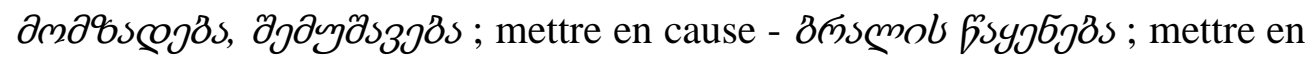

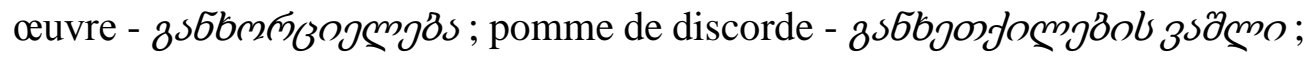

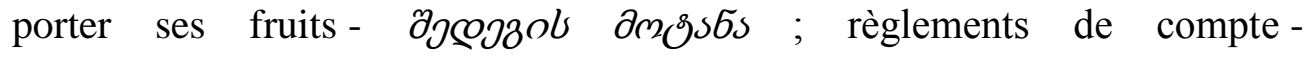

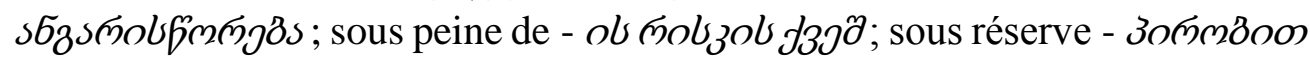

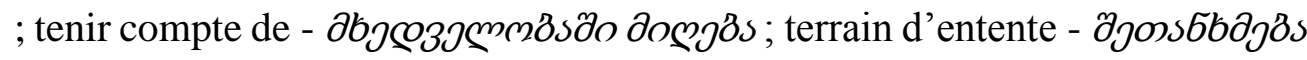

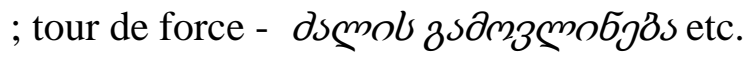

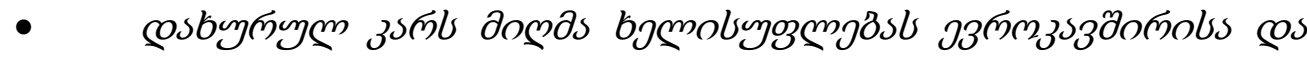

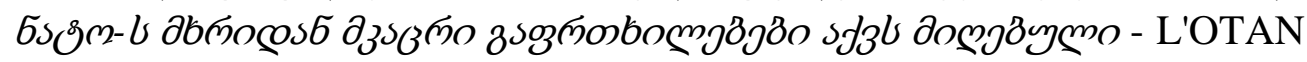
et L'UE ont sévèrement mis en garde le gouvernement géorgien à huis clos

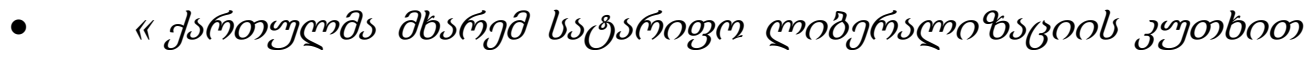

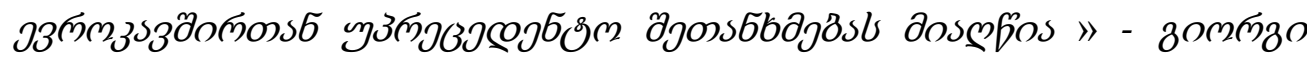
ззопозьдзомо - Selon le ministre de l'Economie et du Développement durable, la Géorgie a conclu avec l'UE un accord sans précédent en matière de libéralisation tarifaire

- Ukraine: l'accord de Genève reste lettre morte - y3mso6ol

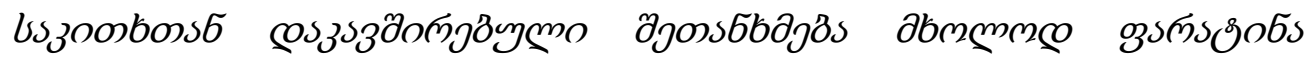
jussmpoos 
- De quelle marge de manœuvre dispose le gouvernement face à

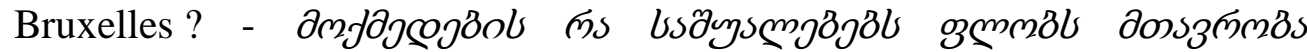

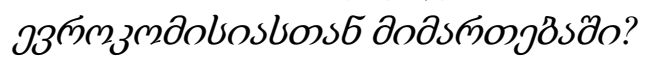

Nombreux sont les cas, où les connaissances culturelles se caractérise par un certain taux d'universalité et les phraséologies de deux langues sont composées de mêmes constituants lexicaux en français et en géorgien :

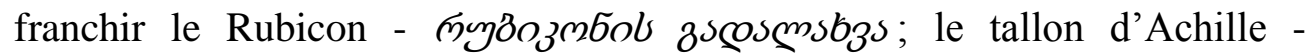
sfomjzlol jylumo; le fil d'Ariane - sॅoscobjl dsgo; le travail de Sisyphe

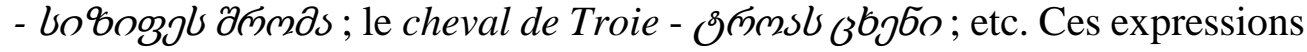
qui sont universellement connues sont largement utilisées dans le discours journalistique :

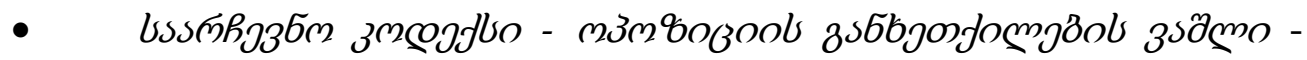
Géorgie : le code électoral constitue une pomme de discorde entre les partis d'opposition

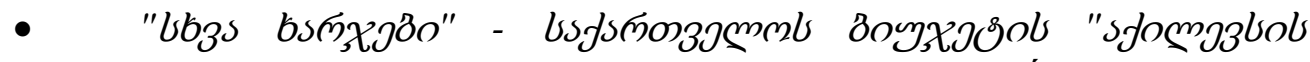
fylmo" - D'autres dépenses- talon d'Achille du budget de l'État géorgien.

Pour pouvoir percevoir correctement les titres des articles politiques il faut pouvoir reconnaître les expressions phraséologiques, connaître leur signification et leur valeur afin de ne pas percevoir le sens de cette expressions d'une manière littérale qui pourrait induire le lecteur en erreur et rendre le titre de l'article incompréhensible et donc pas intéressant. Pour comprendre le sens de certaines expressions phraséologiques, il faut utiliser non seulement les connaissances linguistiques, mais également les connaissances extralinguistiques du fait que la somme des significations des éléments des expressions phraséologiques ne permet pas l'appréhension du sens.

- $\quad 3$ ème couleuvre à avaler en 3 jours pour les électeurs de Hollande -

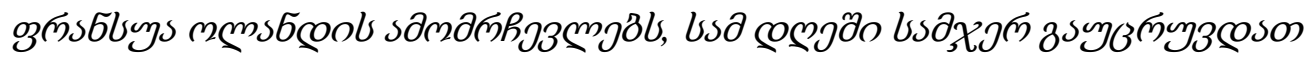
одวณัo.

L'étude de la presse française et géorgienne nous permet de constater que le discours journalistique a recours à des expressions phraséologiques neutres et stylistiquement marquées. Les expressions dits neutres ne sont pas stylistiquement marquées et se transforment le plus souvent en clichés, par ex.

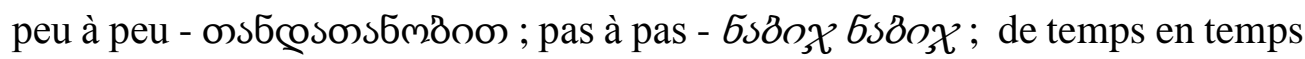

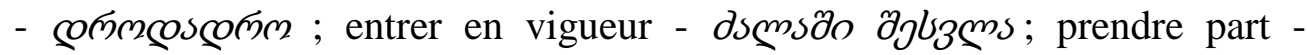

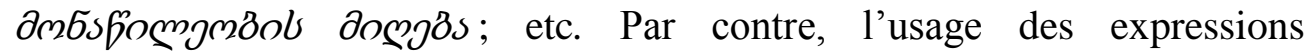
stylistiquement marquées est un procédé régulier : donner le feu vert - $\partial 63\lrcorner \sigma \partial$

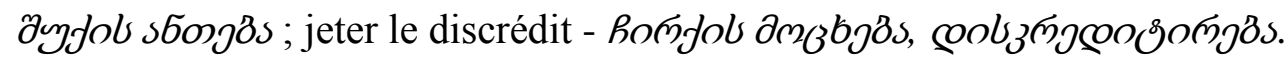


- Le Syndicat de la magistrature accuse Sarkozy de « jeter le discrédit»

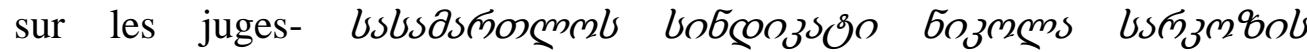

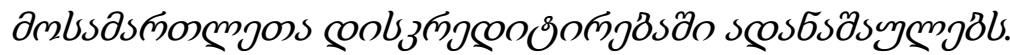

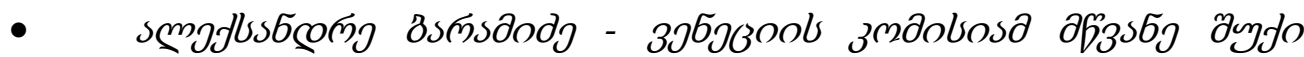

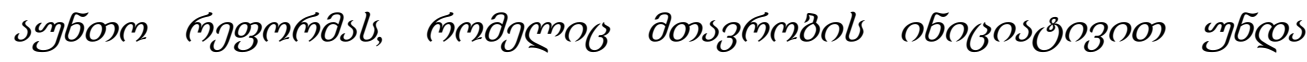

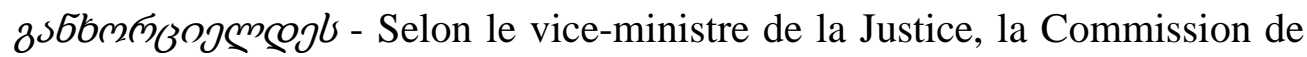
Venise donne le feu vert à la mise en œuvre des réformes initiées par le gouvernement géorgien

Dans le discours journalistique français on utilise assez souvent les expressions phraséologiques avec le composant somatique tel que : la main ; le pied ; l'oreille ; la tête ; l'épaule ; le coude ; etc. p.ex. avoir du sang sur les

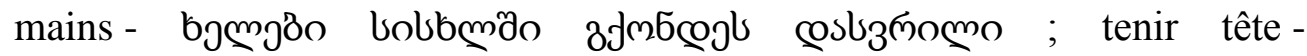

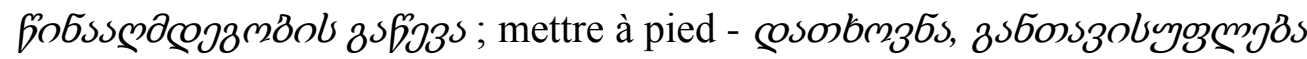

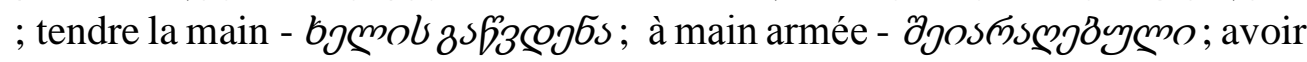

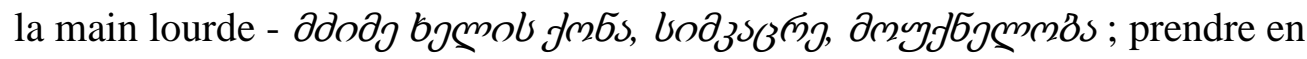

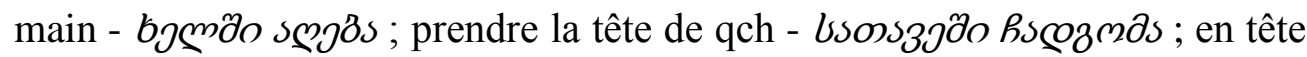

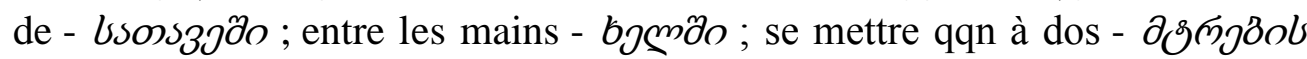

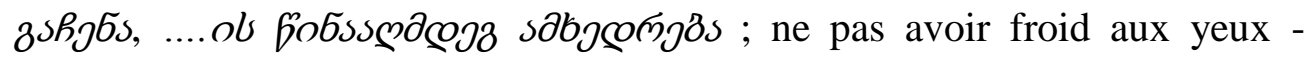

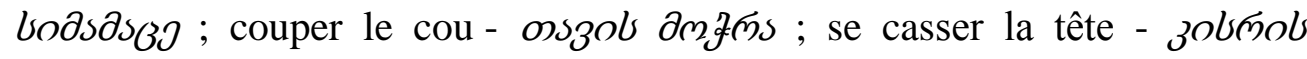

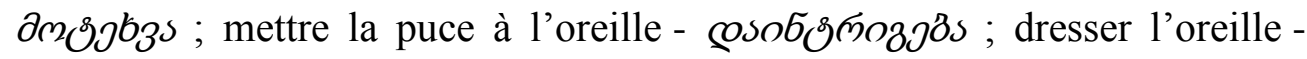

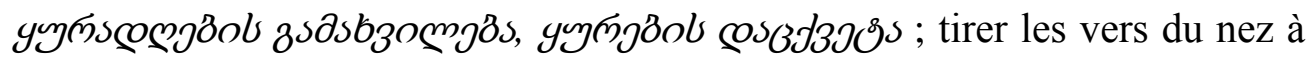

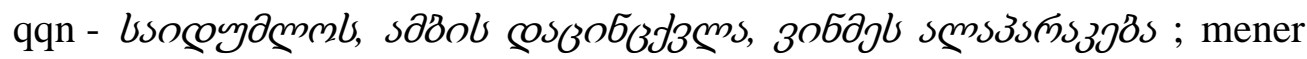

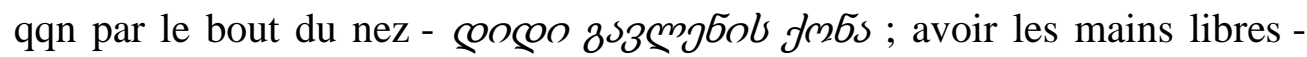

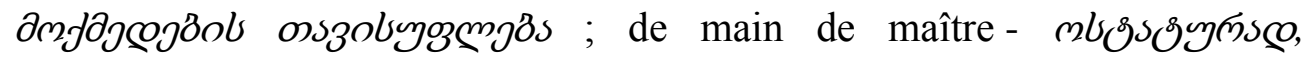

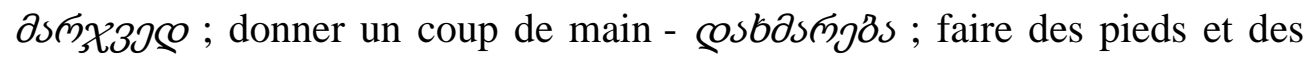

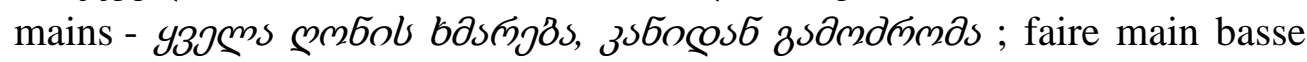

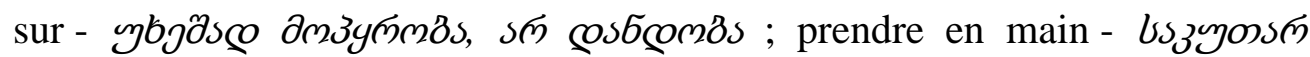

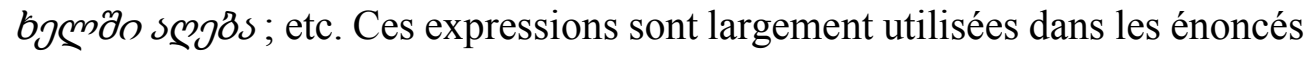
intitulants.

- A Washington, Fabius tient tête aux Américains - $35 \not \partial o 6 z \mathcal{O} m 6 \partial 0$

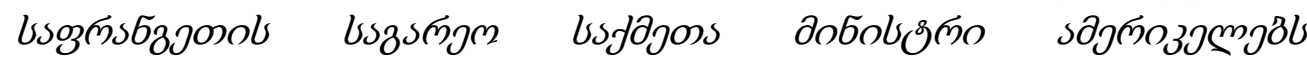
cosyzonolзongs.

- Kosovo : le parti du premier ministre pro-européen en tête des

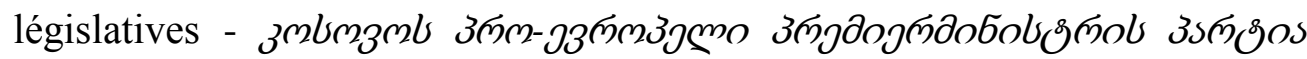

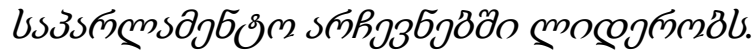

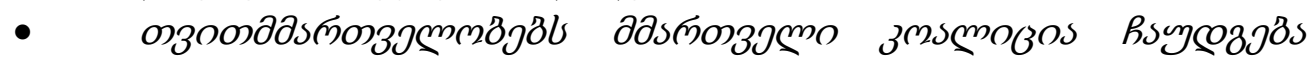
usons 3 Jo - Le parti au pouvoir va prendre la tête des collectivités locales. 
Lors de notre étude, nous avons pu constater que la presse utilise les unités phraséologiques exprimant une attitude négative, ironique ou bien au contraire positive vis-à-vis des personnes ou des événements qui se produisent : tourner le dos à qqn - \&yyrabol gyd(303s ; traîner dans la boue -

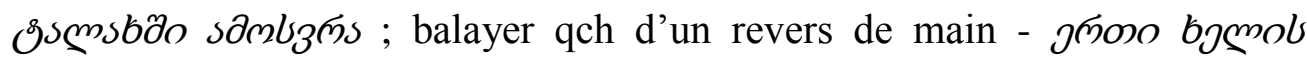

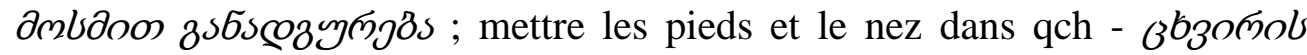
Rsymozs ; jeter la lumière sur... - Gsongngol $\partial m g \jmath 6 s$; vivre en harmonie avec

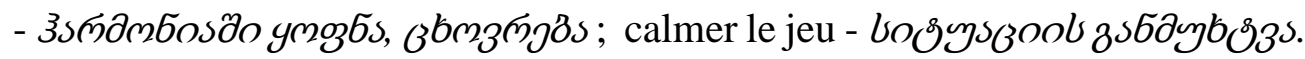
- $\quad$ La télé iranienne traîne dans la boue la femme qui a dévoilé l'Iran -

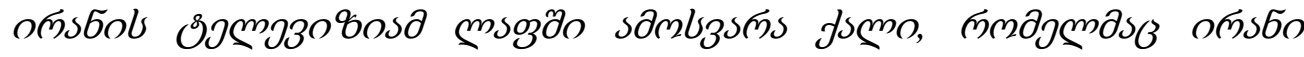
sabons.

- $\quad$ Comment Sarkozy fourre son nez dans les affaires du Monde - бмдмп

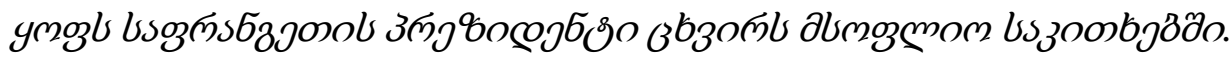

- amlzmzol ammszsñzjojzas usazzocomzmjzol luzoobl

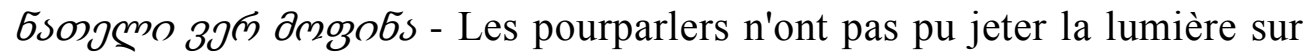
les question de la paix.

En fait, il est difficile de déterminer exactement le nombre d'expressions idiomatiques dans une langue car conformément aux besoins quotidiens les nouvelles structures apparaissent. En plus, suite à l'intensification de la communication et à la diffusion instantanée de l'information, certaines unités phraséologiques sont empruntées à d'autres

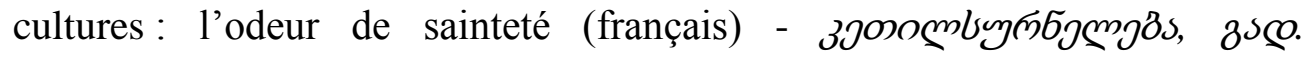
cosossbgos ; fumer le calumet de la paix/enterrer la hache de guerre [amérindien] - જூgrozว 85 ; jeter le bébé / l'enfant avec l'eau du bain [anglais]

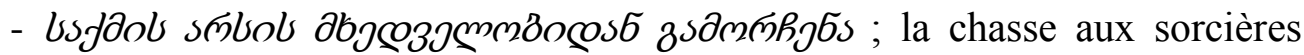

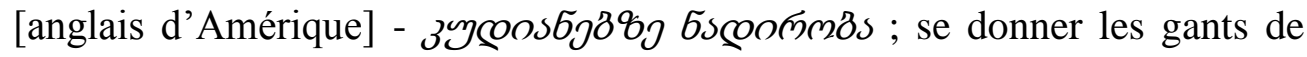
qqch - дoozolyzs, bszyonsm ossz face - odoxol coszsmz3s / zscosm

- $\quad$ Guéant "observe" que Sarkozy "a l'air de revenir en odeur de sainteté"

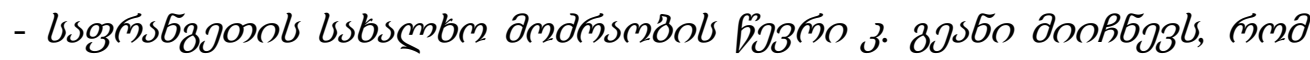

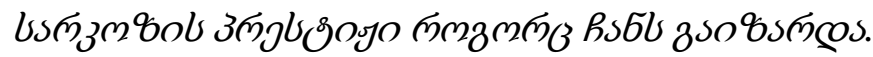

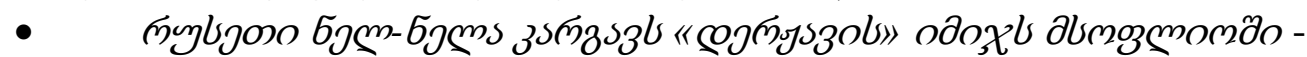
La Russie perd peu à peu l'image de la «derjava dans le monde / l'image de la puissance mondiale 
- $\quad$ Cohésion sociale: Des communautés ivoiriennes "fument le calumet

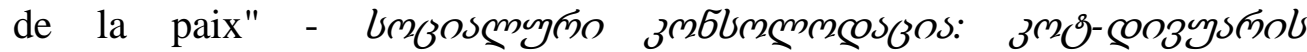

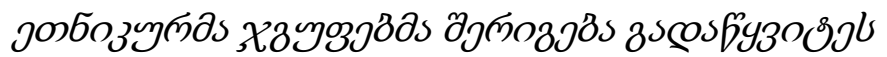

Dans la presse, on utilise très souvent les expressions idiomatiques avec les chiffres: $\partial$ gbyon $3^{m}$ mmbs - la cinquième colonne ; le quatrième

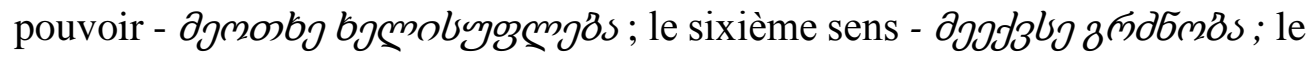

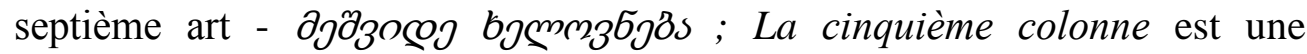
expression d'origine espagnole. Elle est entrée dans le vocabulaire courant dans diverses langues. Par extension, l'expression désigne tout groupement agissant dans l'ombre pour saper de l'intérieur une organisation ou un État. Cette expression est utilisée assez souvent par les journalistes géorgiens en quête constante d'un titre choc. Les expressions idiomatiques avec les chiffres sont également employées dans la presse française :

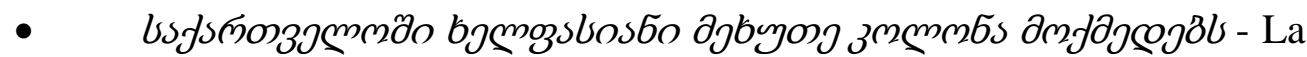
cinquième colonne bien rémunérée opère sur le territoire géorgien.

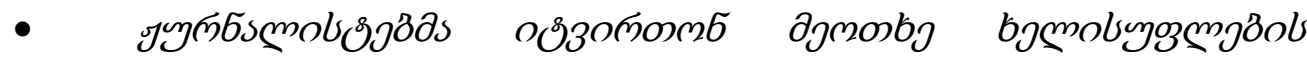

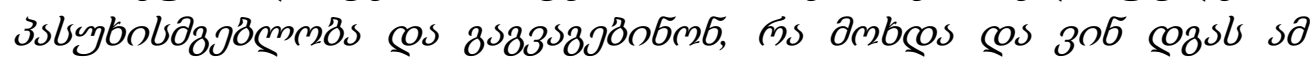
y3วलмsoुnol y3s6 - Les journalistes devraient assumer la responsabilité du quatrième pouvoir et nous expliquer ce qui s'est passé et qui sont derrière ses événements.

- Le septième art au service de la politique -

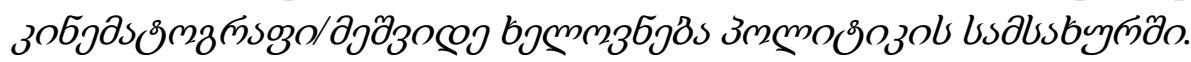

La presse française et géorgienne abondent d'expressions idiomatiques

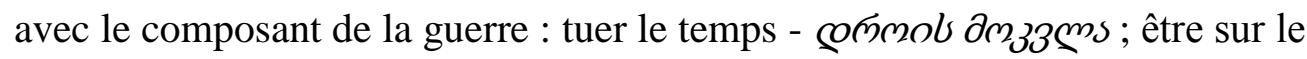

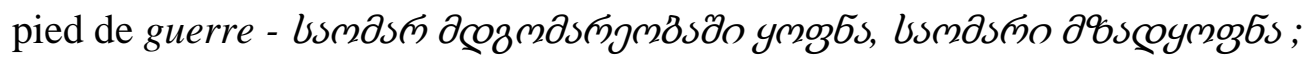

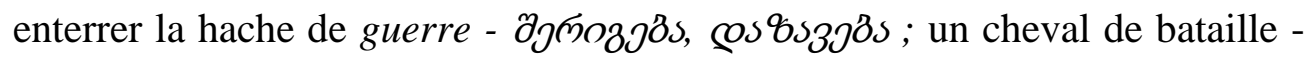

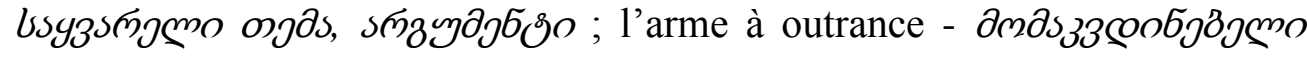

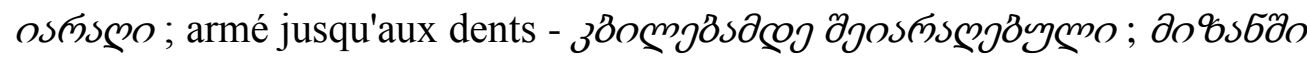

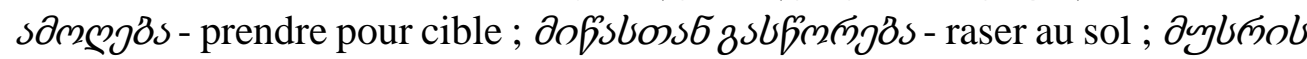

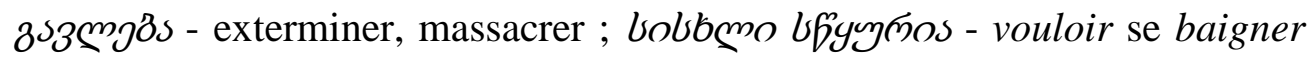

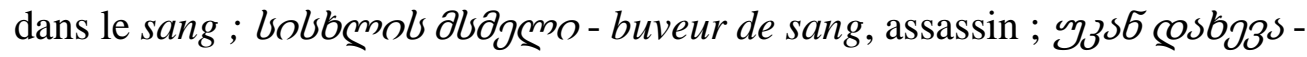
tourner casaque, changer d'avis ; gynnobdojzol osmsmo - arme de vengeance.

- Logo: le Front de Gauche enterre la hache de guerre - $\partial \jmath \partial s_{0} b j 6 \jmath$

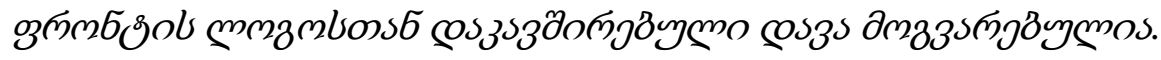

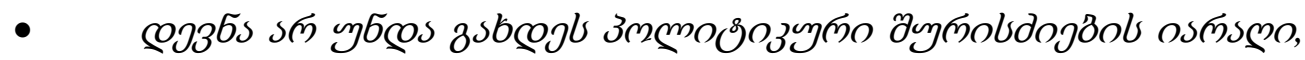

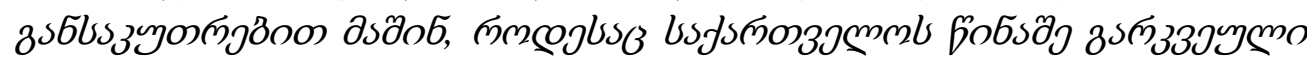

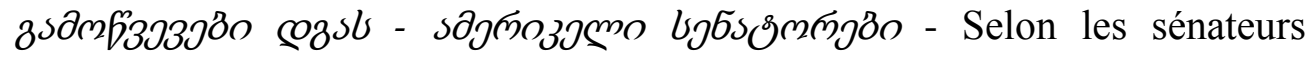


américains, en Géorgie qui devrait faire face à certains défis, la poursuite des anciennes autorités géorgiennes ne devrait pas devenir une arme de vengeance politique.

- EUROPE/UKRAINE-RUSSIE / C'est reparti comme en 14? -

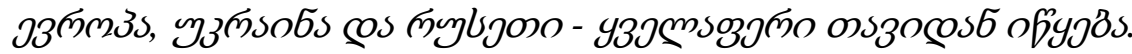

La dernière expression fait allusion à la guerre de 14-18 et à l'enthousiasme avec lequel les soldats sont partis faire une guerre annoncée comme courte et facile. L'expression sous-entend donc une certaine naïveté alors que les circonstances ne s'y prêtent pas.

Le discours journalistique français recourent assez souvent aux expressions idiomatiques avec des couleurs : donner carte blanche à quelqu'un - 3$\lrcorner \curvearrowleft \delta-\partial \mathrm{m} s \sigma \partial o b \partial \rho(\partial \partial s$; faire travailler sa matière grise -

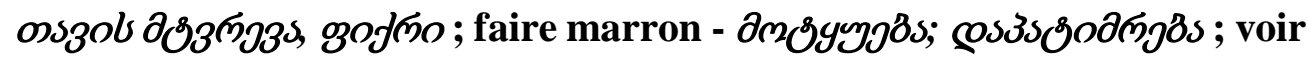

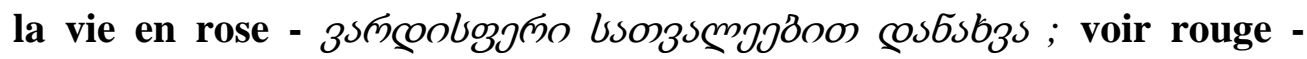

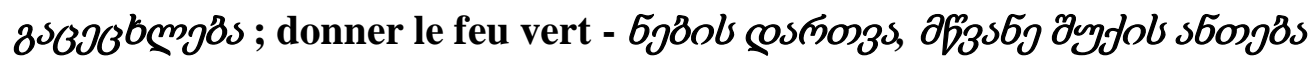
; être blanc comme neige - oym ycos6sasynmm; broyer du noir -

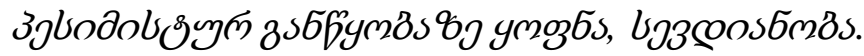

- Le gouvernement canadien veut donner carte blanche aux forces de

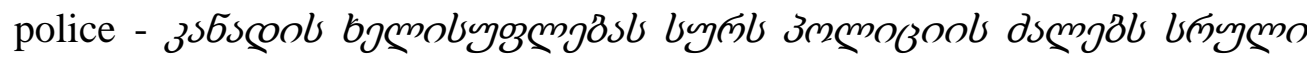
onszolyozmgds dos6o zal.

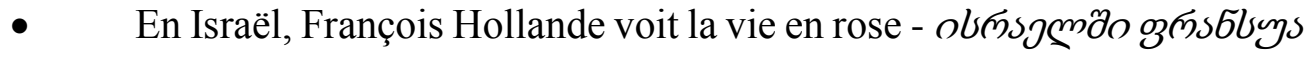

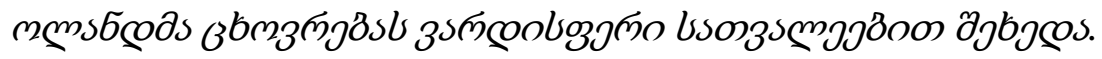

- Dans un Athènes en grève, Socrate voit rouge - zsozogym sonj6zo, bớyszos yzocoynglsço cosdszymos.

- Bruxelles broie du noir pour l'économie française - Вஙoylggno

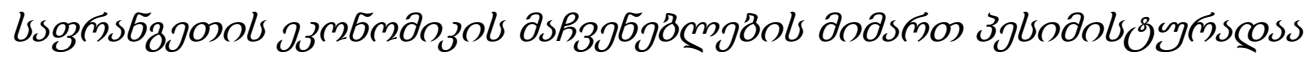
zsббумдомо.

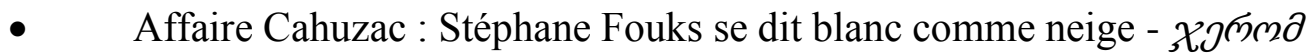

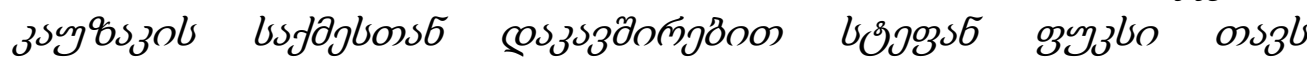

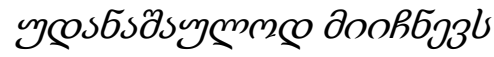

\section{Conclusion}

La phraséologie évolue rapidement. Certaines unités phraséologiques disparaissent assez vite, d'autres deviennent une partie intégrante de la langue. Il est difficile de déterminer exactement le nombre d'expressions idiomatiques dans une langue. La maîtrise de la phraséologie est une part importante de la compétence journalistique du fait que les textes journalistiques regorgent d'unités phraséologiques. Très variées sur le plan sémantique, les locutions 
idiomatiques enrichissent effectivement le discours et le rendent beaucoup plus expressif.

\section{References:}

BALly, Charles, Traité de stylistique française, Paris, Librairie Georg, 1951. CAPRA, Antonella, Traduttore traditore : de la possibilité de traduire les expressions figées en littérature http://revuesshs.ubourgogne.fr/textes\&contextes/document.php?id=1303\&format=print (consulté le 23.08.2014)

DuCROT, Oswald, «Présupposés et sous-entendus », dans Langue française 4, Paris : Larousse, 1969

LE BEL, Edith, Traduire la phraséologie : réflexions méthodologiques et études de cas dialnet.unirioja.es/descarga/articulo/2254286.pdf (consulté le 15.07.2014).

Gross, Gaston, Les expressions figées en français : noms composés et autres locutions, Paris, Ophrys, 1996.

LE GUERN, Michel, Sémantique de la métaphore et de la métonymie. Paris, Larousse, Collection Langue et Langage, 1973.

PAVEL, Silvia, «La phraséologie en langue de spécialité. Méthodologie de consignation dans les vocabulaires terminologiques », dans Terminologies nouvelles, 10, pp. 67-82, 1993. 